\title{
Comparing how anxiety is experienced between white and minority patients after local therapy for prostate cancer: A hypothesis-generating pilot study
}

\author{
Channa Amarasekera; Vincent Wong; David Victorson; James Burns; David Cella; Shilajit \\ Kundu \\ Northwestern University Feinberg School of Medicine, Chicago, IL, United States
}

Cite as: Can Urol Assoc J 2018 July 24; Epub ahead of print. http://dx.doi.org/10.5489/cuaj.5373

Published online July 24, 2018

$* * *$

\section{Introduction}

Men diagnosed with prostate cancer bear the burden of a cancer diagnosis, compounded by the stress of choosing from a variety of treatment options. Treatment may have associated side effects including erectile, urinary, and bowel dysfunction, among others, all of which may lead to heightened levels of anxiety in patients. (1) While disparities in cancer specific and surgical outcomes have been studied in men of racially diverse backgrounds with prostate cancer, how a minority racial identity influences perceptions of anxiety with prostate cancer is not well delineated. (2) Thus, our study aims to evaluate differences in psychosocial effects of prostate cancer and its treatment between non-Hispanic Whites and minority groups (African Americans and Hispanic men).

\section{Methods}

We enrolled 105 men from the Department of Urology at Northwestern Hospital with clinically localized prostate cancer who had yet to receive primary treatment (surgery, radiation, or active surveillance) in an internet based self-reported longitudinal study. They completed validated instruments measuring anxiety as well as sexual and bladder function. The International Index of Erectile Function (IIEF) scale was used to assess erectile function. (3) For anxiety and bladder symptoms, they completed the respective Surgical Outcomes Measurement System (SOMS) scales. This instrument is an extension of the Patient Reported Outcomes Measurement Information System (PROMIS), a National Institutes of Health funded validated measurement system used to make accurate and efficient assessments of multiple health domains. SOMS was developed using the same methodology used to develop PROMIS and whenever possible PROMIS questions were incorporated into SOMS. $(4,5)$ The assessments were completed prior to treatment for a baseline assessment, then repeated at 1, 3, 6, and 12 months after treatment. We focused this analysis on the 70 patients who chose local therapy (surgery or radiation 
therapy) for treatment, with 55 of these men being non-Hispanic white and 15 being African American or Hispanic. The effects of prostate cancer and local treatment were assessed using multivariate tests controlling for other social factors like income, marital status, and education. Linear mixed models were used to examine changes in self-reported measures at enrollment (pre-treatment) and at each post-treatment follow-up assessment.

\section{Results}

Erectile function, bladder function and anxiety scores for non-Hispanic White men compared to African American and Hispanic men are shown in the Figure. A decrease in erectile and bladder function scores indicates a worse outcome, while a decrease in anxiety scores indicates improvement. Despite significant decreases in erectile and bladder function from baseline $(\mathrm{P}<.001)$, both groups reported an improvement in anxiety post-treatment $(\mathrm{P}<0.01$ for both). When controlling for differences in income, marital status, education, and improvements in urinary score, the impact of treatment on anxiety remained significant $(\mathrm{P}<0.05)$ at 3,6 , and 12 months.

Previous work has found that the minimally clinically important difference for anxiety scores, defined as "a difference in score that is large enough to have implications for a patient's treatment or care" to be 3.0-4.5 points on the T-score. (6) Anxiety score reductions for both groups in our study exceeded this range. After 12 months, anxiety for Non-Hispanic White men decreased by 4.6 points $(\mathrm{P}<0.01)$ and anxiety scores for minority men decreased by 7.9 points $(\mathrm{P}<.001)$, with similar IIEF scores in each group at 12 months. Additionally, at 12 months, both groups had anxiety T-scores that were lower than the average anxiety score of the general population without a cancer diagnosis, which is represented by a T-score of 50

\section{Discussion}

The diagnosis of prostate cancer in a man who elects for screening can provoke a great deal of anxiety and have a profound impact on his psychosocial health. Studies have found between $26.6 \%$ to $38 \%$ of men reporting psychological distress after being diagnosed with prostate cancer. $(7,8)$ It has also been found that prostate cancer treatment reduces psychological distress and anxiety both in the short and the long term. $(9,10)$ For example, Korfage et al followed 299 men with localized prostate cancer over 5 years and had them complete assessments of anxiety and depression prior to treatment and at 6 months, 12 months, and 5 years follow up. They found sustained statistically significant reductions in anxiety in men who had treatment for their prostate cancer.

However, these studies have not examined differences in how prostate cancer affects anxiety and mental well-being of different racial groups. The present study found significant reductions in anxiety after prostate cancer treatment in men who chose local therapy, despite a deterioration in erectile and bladder function, with a trend toward greater reductions in anxiety in minority men compared to non-Hispanic White men. Though validated instruments to measure 
anxiety exist, anxiety assessments are not routinely performed in the clinical setting prior to prostate cancer treatment. Our study highlights the importance of gauging a man's psychosocial outlook when he chooses treatment, especially since racial identity may have an impact on how anxiety is experienced with treatment of prostate cancer. However, it should be stressed that the work presented here represents a preliminary hypothesis generating study, and our findings need to be corroborated with larger cohorts of similar patients. 


\section{References}

1. Shrader-Bogen Cheryl L., Kjellberg Janice L., McPherson Carol P., Murray Charles L. Quality of life and treatment outcomes. Cancer. 2000 Sep 29;79(10):1977-86.

2. Haider AH, Scott VK, Rehman KA, Velopulos C, Bentley JM, Cornwell EE, et al. Racial disparities in surgical care and outcomes in the United States: a comprehensive review of patient, provider, and systemic factors. J Am Coll Surg. 2013 Mar;216(3):482-492.e12.

3. Rosen RC, Riley A, Wagner G, Osterloh IH, Kirkpatrick J, Mishra A. The international index of erectile function (IIEF): a multidimensional scale for assessment of erectile dysfunction. Urology. 1997 Jun;49(6):822-30.

4. Cella D, Yount S, Rothrock N, Gershon R, Cook K, Reeve B, et al. The Patient-Reported Outcomes Measurement Information System (PROMIS): progress of an NIH Roadmap cooperative group during its first two years. Med Care. 2007 May;45(5 Suppl 1):S3-11.

5. Zapf M, Denham W, Barrera E, Butt Z, Carbray J, Wang C, et al. Patient-centered outcomes after laparoscopic cholecystectomy. Surg Endosc Interv Tech. 2013 Dec 1;27(12):4491-8.

6. Yost KJ, Eton DT, Garcia SF, Cella D. Minimally important differences were estimated for six Patient-Reported Outcomes Measurement Information System-Cancer scales in advanced-stage cancer patients. J Clin Epidemiol. 2011 May;64(5):507-16.

7. Balderson N, Towell T. The prevalence and predictors of psychological distress in men with prostate cancer who are seeking support. Br J Health Psychol. 2003 May;8(Pt 2):125-34.

8. Carlson LE, Angen M, Cullum J, Goodey E, Koopmans J, Lamont L, et al. High levels of untreated distress and fatigue in cancer patients. Br J Cancer. 2004 Jun 14;90(12):2297304.

9. Korfage IJ, Essink-Bot M-L, Janssens ACJW, Schröder FH, Koning HJ de. Anxiety and depression after prostate cancer diagnosis and treatment: 5-year follow-up. Br J Cancer. 2006 Apr;94(8):1093-8.

10. Litwin Mark S., Lubeck Deborah P., Spitalny G. Mark, Henning James M., Carroll Peter R. Mental health in men treated for early stage prostate carcinoma. Cancer. 2002 Jun 28;95(1):54-60. 
Figures and Tables

Fig. 1. Anxiety, erectile function, and bladder symptoms before and after local therapy for prostate cancer. As asterisk indicates a significant change from baseline.
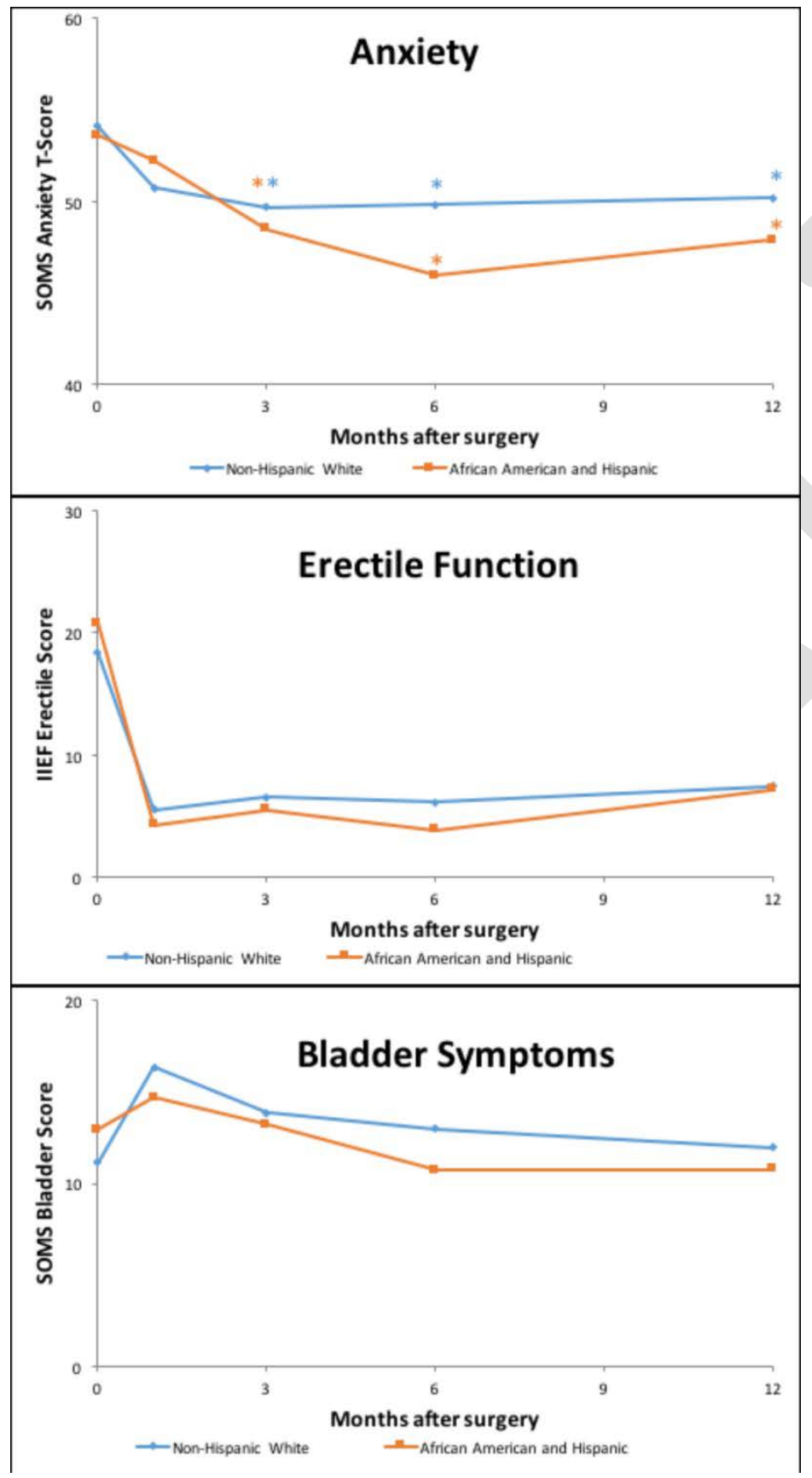